\title{
Circuit
}

Musiques contemporaines

\section{Les oeuvres de Mario Bertoncini}

\section{John Rea}

Volume 15, numéro 1, 2004

Interpréter la musique (d')aujourd'hui

URI : https://id.erudit.org/iderudit/902348ar

DOI : https://doi.org/10.7202/902348ar

Aller au sommaire du numéro

Éditeur(s)

Les Presses de l'Université de Montréal

ISSN

1183-1693 (imprimé)

1488-9692 (numérique)

Découvrir la revue

Citer ce document

Rea, J. (2004). Les oeuvres de Mario Bertoncini. Circuit, 15(1), 105-108.

https://doi.org/10.7202/902348ar

Ce document est protégé par la loi sur le droit d'auteur. L'utilisation des services d'Érudit (y compris la reproduction) est assujettie à sa politique d'utilisation que vous pouvez consulter en ligne.

https://apropos.erudit.org/fr/usagers/politique-dutilisation/
Cet article est diffusé et préservé par Érudit.

Érudit est un consortium interuniversitaire sans but lucratif composé de l'Université de Montréal, l'Université Laval et l'Université du Québec à Montréal. Il a pour mission la promotion et la valorisation de la recherche. https://www.erudit.org/fr/ 


\title{
Les œuvres
}

\section{de Mario Bertoncini}

\author{
PAR JOHN REA
}

\section{CEUVRES MUSICALES}

Elementi di forma (2000-2001), violoncelle préparé et traitement électronique.

II Cimitero degli Elefanti (Le cimetière des éléphants) (1979; nouvelle version : 2000), pianos et traitement électronique.

Exercice (1999), danseuse et environnement sonore interactif.

Suite "colori" (1999), piano préparé.

La favola d'Aracne (La fable d'Arachnê) (1999; non réalisée), projet de théâtre musical pour harpe éolienne, tam-tam, récitant, chœur, deux ballerines et projections.

Aracne (Arachnê) (1999), harpe éolienne.

Fuochi (Feux, Foyers) (in memoriam Angela Loij) (1998-1999), huit instruments à vent et disque compact à huit pistes avec huit interprètes.

Sinfonia "dei respiri" (Symphonie des souffles) (1997), neuf instruments.

Solo aus Klavierquartett (Solo, tiré du quatuor avec piano) (19931994), câble suspendu et cinq résonateurs [portant aussi le nom de Quatuor à cordes, $\left.n^{\circ} 4\right]$.

Streichquartett Nr. 2 (1993), quatuor à cordes avec sourdines à barre.

Ricercare sul tema di "Chanson" (1992), deux groupes de harpes éoliennes et deux interprètes [voir plus bas, Chanson pour instruments à vent].

Streichquartett Nr. 1 (Quatuor à cordes, $n^{\circ} 1$ ) "Die Lyra des Äolus" (1990-1992), quatre gongs éoliens et quatre interprètes.

Astrolabium (1990, non réalisée), harpe éolienne, danseuse et choréphone.
Elisaveta Bam (1984), musique de scène pour cinq instruments, harpe éolienne, orgue de Barbarie et quatre voix d'hommes.

Pagine per Dieter (1983), pour le compositeur D. Schnebel (interprète) et traitement électronique.

Alleluia (1982), huit gongs japonais et mécanisme d'un piano à queue.

Bheri (1973-1981), sept gongs, moteurs et projection d'ombres, un interprète.

Venti (Vents, Vingt) (1980-1982), installation éolienne pour vingt harpes éoliennes, quarante interprètes et deux chefs d'orchestre. An American Dream (1974), piano préparé avec petits moteurs. Pavana (1974), flute, hautbois, clarinette, basson, cor et piano. Focus (1974), danseur, environnement sonore et lumineux.

Chanson pour instruments à vent (1974), harpes éoliennes et gongs éoliens, un interprète.

Vele (Voiles, Toiles) (1974), trois groupes de harpes éoliennes et trois vocalistes.

Chain Reaction (1973), harpes éoliennes, gongs éoliens et environnement lumineux (réalisée avec le peintre cinétique, Peter Sedgley).

Scratch-a-matic (1971), piano et moteurs à courant continu. Suite dal Balletto (1970), orchestre de chambre.

Tempi di prova (Temps de répétition) (1970), six interprètes ou six groupes d'interprètes.

Mariolina (1969-1970), clavecin.

A New Beggar's Opera (1969), piano et ensemble variable. 
Tre fogli x 4 (Trois feuilles x 4) (1969), percussions jouées par des enfants.

Spazio-Tempo (Espace-Temps) (1967-1969), œuvre audiovisuelle pour mimes-danseurs, instrumentistes, diapositives et traitement électronique en direct.

Illegonda (1968), soprano et environnement musical.

Epitaffio in memoria di un concerto (1968), trois sources sonores et traitement électronique en direct.

Cifre (Chiffres, Sommes) (1964-1967), piano préparé avec un ou plusieurs interprètes.

Tune (1965), cymbales suspendues.

Quodlibet (1964), alto, violoncelle, contrebasse et percussion.

Sei pezzi per orchestra (1962).

\section{GEUVRES LITTÉRAIRES}

(2003), "Äolsharfen und andere nutzlose Dinge" [Dialogue $n^{\circ} 8$ ] MusikTexte, $n^{\circ}$ 96, p. 46-57.

(1996), "One Day at the Centre of Earth" [Dialogue n 6, Jour 4] Notes of the Network for International Cooperation in the Arts (Universität der Künste, Berlin), sans pagination.

(1990), Sonetti scelti [190 sonnets choisis], Berlin, Æolus Verlag, $208 p$

(1985), "Note per un teatro della realtà " [Dialogue $n^{\circ} 3$; Notes pour un théâtre de la réalité], Décalage (Sonorità Prospettiche), $n^{0} 13$, p. 21-28.

(1979), "Proposta di construire un istituto sperimentale di ricerca sul suono", MOLLIA, M. (s. la dir. de), Autobiografia della musica contemporanea, Cosenza, p. 81-89.

(1978-), Sonetti [plus de 450 sonnets écrits en dialecte romain (romanesco), inédits à l'exception de 190 poèmes].

(1976-2000), "Ragionamenti musicali in forma di dialogo"

[Raisonnements musicaux en forme de dialogue],

Dialogue no 1 (1998), Almost a Preface, 3 p. man.;

Dialogue $n^{\circ} 2$ (1976), M. Bertoncini : Entretien [entretien accordé à Raymond Gervais, revu et corrigé par Bertoncini; voir bibliographie];
Dialogue $n^{\circ} 3$ (1981), Note per un teatro della realtà (voir CEuvres littêraires);

Dialogue ${ }^{\circ} 4$ (1988-1989), In rotta verso il duemila : Conversazion televisive in tre giornate - Giornata Prima : Una Strega, Giornato Seconda : II Selvaggio, Giornata Terza : II Successo [En route vers I'an 2000 : Conversations télévisuelles en trois journées - Jour 1 , Une Sorcière; Jour 2, Le Sauvage; Jour 3, Le Succès], 128 p. man. (inédit);

Dialogue $n^{\circ} 5$ (1995), Una sera di luglio nei giardini di Accademo, [Une soirée de juillet dans les jardins d'Akadémos], 14 p. man. (inédit);

Dialogue $n^{\circ} 6$ (1996), Sette giorni al centro della terra [Sept jours au centre de la terre] : Jour 1, da Tel Aviv a Gerusalemme, Jour 2, Verso Masada, Jour 3, Masada, Jour 4, L'Accademia, il campus, i colleghi (voir CEuvres littéraires), Jour 5, II Concerto, Jour 6, Gerusalemme, Jour 7, II Museo, 21 p. man. (inédit);

Dialogue $n^{\circ} 7$ (1998-2003), Dell'imitazione [Sur l'imitation], 58 p. man. (inédit);

Dialogue $n^{\circ} 8$ (1999-2000), Arpe eolie e altre cose inutili [Harpes éoliennes et autres choses inutiles] (voir Cuuvres littéraires);

Dialogue $n^{\circ} 9$ (2000), II sogno della complicazione ovvero la complicazione del sogno [Le rêve de la complication ou la complication du rêve], 45 p. man. (inédit).

\section{DISCOGRAPHIE}

CAGE, J. (2001), Sonatas and Interludes. Mario Bertoncini, piano préparé. DAAD et Édition RZ, Parallele CD 20001, "ZeitenwechselJohn Cage".

BertonCINI, M. (1989), Cifre [versions pour deux pianos préparés et pour trois pianos préparés], Four systems [E. Brown], Cartridge Music [J. Cage]. Édition RZ LP-1002, "Mario Bertoncini".

BERTONCINI, M. (1972), Tune [et œuvres de J. Cage, L. Harrison] Black Earth Percussion Group. Opus One LP 22-2800.

(1967), Lip Service, RKBA-1675: Take One, Perfect Union, Side OneBand Four, Springs Quartet, Sunrise, Conversations, Waves, II Gruppo d'Improwisazione Nuova Consonanza [ M. Bertoncini, F. Evangelisti, R. Kayn, E. Morricone, J. Heineman, F. Rzewski, I. Vandor ]. RCA Victor LP LSP-3846, "The Private Sea of Dreams". 


\section{BIBLIOGRAPHIE}

ANDERSON, C. (2003), "Dialoge und andere "nutzlose" Texte, Anmerkungen zu den Schriften von Mario Bertoncini", MusikTexte, $n^{\circ} 96$, p. $44-45$.

BertonCINI, M. (1968), "Cifre, for piano and prepared piano", Source: Music of the Avant Garde (1967-1972), unité 4, vol. 2, nº 2 , p. 7-9.

BORIO, G. (1999), "Bertoncini, Mario", Musik im Geschichte und Gegenwart, Personenteil, vol. 2, p. 1471-1473.

BORIO, G. (1994), "Mario Bertoncini : Porträt eines radikalen Neueres", Montags Musik : Mario Bertoncini (Berlin).

Borıo, G. (1992), "Klang als Prozeß: Die Komponisten um "Nuova Consonanza" ", Angermann, K. (s. la dir. de), Giacinto Scelsi, Taunus, Hofheim Verlag, p. 11-25.
Bortolotto, M. (1970), "Colloquio con M. Bertoncini", Lo spettatore musicale, $\mathrm{n}^{\circ} 3$, p. 20-23.

FRIEDL, R. (2000), "Schlagen, streichen, bürsten, werfen... Mario Bertoncini, der Entdecker des Klavierinnenraums", Neue Zeitschrift für Musik, $n^{\circ} 1$, p. 22-25.

Gervals, R. (1976), "M. Bertoncini : Entretien ", Parachute, n 4, p. 10-15. [Ce texte a été revu et corrigé par Bertoncini, Dialogue $n^{\circ}$ 2.]

IBER, M. (1995), "Cifre (1964-67), Rundfunkmanuskript, Version für 3 Klaviere, Piano Inside-Out Trio (Reinhold Friedl, Stefano Taglietti, Michael Iber)",

http://www.iber-online.de/scripts/cifre/hauptteil_cifre.html, http://www.iber-online.de/scripts/cifre/partitur/hauptteil_partitur.html

SCHNEBEL, D. (2003), "Laudatio", MusikTexte, nº 96, p. 42-43. 


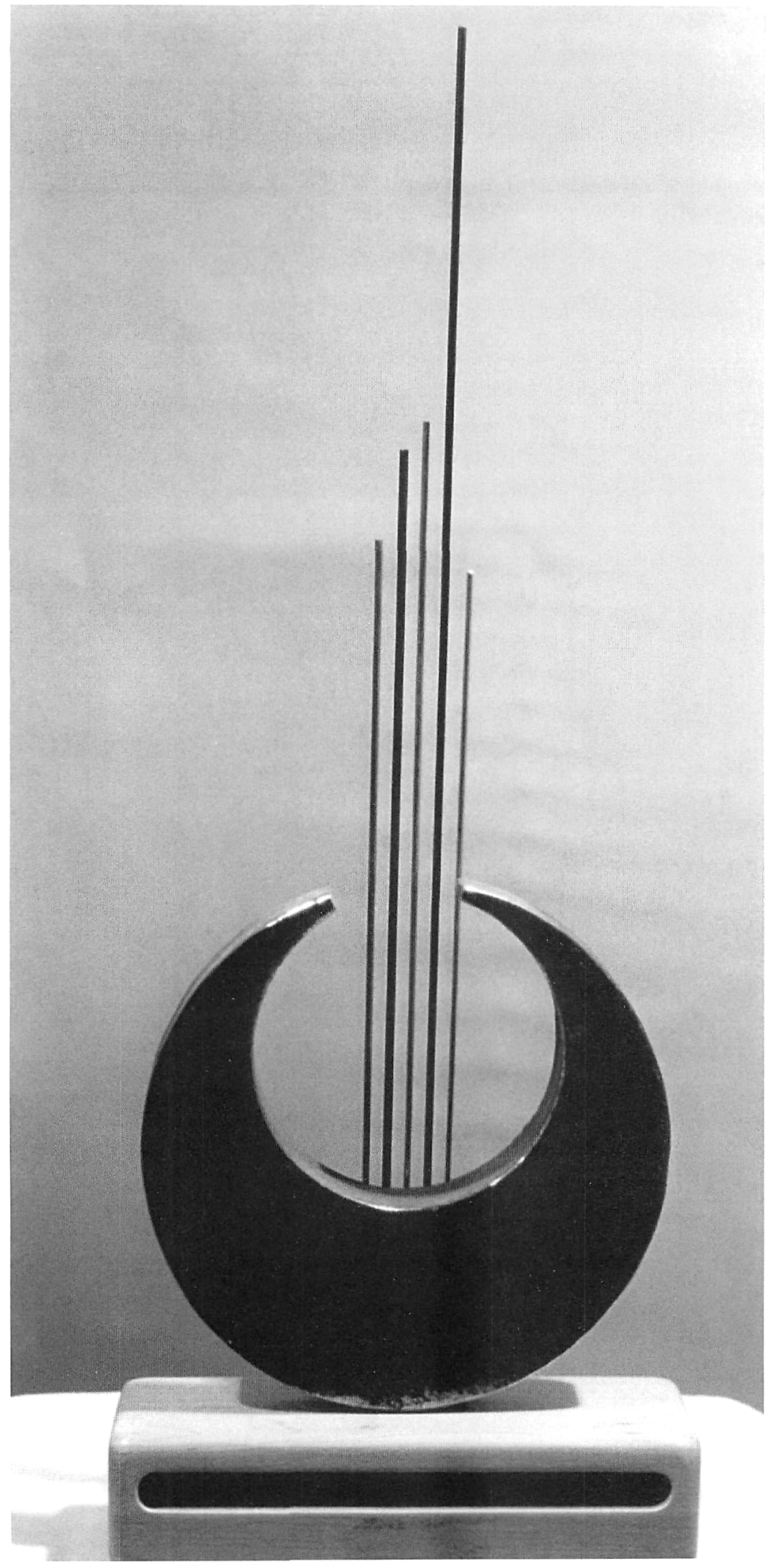

